\title{
Tomada de decisões rápidas: investigação da relação entre múltiplas tarefas e impulsividade
}

\author{
Making quick decisions: an investigation on the relationship between multiple \\ tasks and impulsiveness
}

Toma de decisiones rápidas: investigación de la relación entre múltiples tareas e impulsividad

\author{
Valéria Marcondes Pereira, Doutora \\ Academia da Força Aérea - AFA \\ Pirassununga/SP - Brasil \\ valeria_afa@yahoo.com.br \\ Luiz Maurício de Andrade da Silva, Doutor \\ Academia da Força Aérea - AFA \\ Pirassununga/SP - Brasil \\ Ima28@uol.com.br
}

\section{RESUMO}

Este artigo é parte de um projeto de pesquisa que tem o objetivo de contribuir com o treinamento de pilotos militares, especificamente com o treinamento para decisões rápidas. O ponto de partida da pesquisa é o teste de aptidão para pilotagem militar (TAPMIL), que avalia o potencial de aprendizagem do candidato para a pilotagem militar, com vistas à instrução de voo realizada nos Esquadrões de Instrução Aérea da Academia da Força Aérea (AFA). É um teste psicológico totalmente informatizado, composto por uma bateria de seis testes que avaliam, de maneira isolada ou combinada, aptidões inerentes à pilotagem, como "capacidades cognitivas" (por exemplo, processamento de informação, velocidade e precisão de respostas, memória de trabalho) e capacidade em "tarefas múltiplas" (psicomotoras e cognitivas combinadas) (COSTA, 2010, p. 6). Buscam-se evidências para a atribuição de pesos para as diferentes variáveis intervenientes no problema da aptidão (SILVA et al., 2009), o que nos remete a eleger - até o presente momento - como variável de destaque, a capacidade em execução de múltiplas tarefas. O foco, na variável 'múltiplas tarefas', levou-nos a novas linhas investigativas, como estudos sobre o julgamento humano em decisões, sobretudo aquelas tomadas em ambientes dinâmicos e de grande incerteza (KLEIN, 2009), e a correlação existente entre a capacidade de executar múltiplas tarefas e a impulsividade. Com vistas a continuar essa linha investigativa a respeito da correlação existente entre múltiplas tarefas e impulsividade, o presente trabalho tem por objetivo apresentar os resultados de dois testes realizados com os cadetes da Força Aérea Brasileira, aviadores da turma de 2011, em um total de 60 voluntários. Para a verificação da capacidade de executar múltiplas tarefas, utilizou-se o teste "tudo ao mesmo tempo" (HERCULANO-HOUZEL, 2014). Para o teste da impulsividade, com 45 (quarenta e cinco) voluntários, utilizou-se a Escala de Impulsividade de Barratt - BIS 11.

Palavras-chave: Múltiplas tarefas. Impulsividade. Tomada de decisão. Desempenho.

Recebido / Received / Recibido $22 / 09 / 14$
Aceito / Accepted / Aceptado 22/05/15 


\section{ABSTRACT}

This article is part of a research project that aims at contributing to the training of military pilots, specifically training for quick decisions. The starting point of the research is the aptitude test for military flying (TAPMIL), which assesses the candidate's learning potential for military flying, with a view to flight instruction performed in the Air Instruction Squadrons of the Air Force Academy (AFA). It is a fully computerized psychological test, comprised of a battery of six tests that assess, either alone or in a combined manner, inherent flying skills, such as the "cognitive capacities" (e.g., information processing, speed and accuracy of responses, working memory) and the ability to "multitask" (psychomotor and cognitive combined) (COSTA, 2010, p. 6). Evidence are sought for assigning weights to the different variables intervening in the aptitude problem (SILVA et al., 2009), which brings us to choose - to date - as a featured variable, the ability to run multiple tasks. The focus in the "multitask" variable led us to new investigative lines, such as studies on human judgment in decisions, particularly those taken in dynamic environments and of great uncertainty (KLEIN, 2009), and the correlation between the ability to perform multiple tasks and impulsiveness. In order to continue this investigative line about the correlation between multiple tasks and impulsiveness, this study aims at presenting the results of two tests performed with the Brazilian Air Force cadets, aviators from the class of 2011, a total of 60 (sixty) volunteers. To verify the ability to multitask, the "all at once" test was used (HERCULANOHOUZEL, 2014). For the impulsiveness test, 45 (forty-five) volunteers used the Barratt Impulsiveness Scale - BIS 11.

Keywords: Multitasking. Impulsiveness. Decision-making. Performance.

\section{RESUMEN}

Este artículo forma parte de un proyecto de investigación que tiene el objetivo de contribuir con el entrenamiento de pilotos militares, específicamente con el entrenamiento para decisiones rápidas. El punto de partida de la investigación es la prueba de aptitud para pilotaje militar (TAPMIL), que evalúa el potencial de aprendizaje del candidato para el pilotaje militar, con el objetivo de instrucción de vuelo realizada en los Escuadrones de Instrucción Aérea de la Academia de la Fuerza Aérea (AFA). Es un una prueba psicológica totalmente informatizada, compuesta por una batería de seis testes que evalúan, de manera aislada o combinada, aptitudes inherentes al pilotaje, como "capacidades cognitivas" (por ejemplo, procesamiento de información, velocidad y precisión de respuestas, memoria de trabajo) y capacidad en "tareas múltiples" (psicomotoras y cognitivas combinadas) (COSTA, 2010, p. 6). Se buscan evidencias para atribuir pesos a las diferentes variables intervinientes en el problema de la aptitud (SILVA et al., 2009), lo que nos remite a elegir - hasta el presente momento - como variable de destaque, la capacidad de ejecución de múltiples tareas. El foco, en la variable 'múltiples tareas', nos llevó a nuevas líneas investigativas, como estudios sobre el juicio humano en decisiones, sobre todo aquellas tomadas en ambientes dinámicos y de gran incertidumbre (KLEIN, 2009), y la correlación existente entre la capacidad de ejecutar múltiples tareas y la impulsividad. Pretendiendo continuar esa línea investigativa a respecto de la correlación existente entre múltiples tareas e impulsividad, el presente trabajo tiene como objetivo presentar los resultados de dos testes realizados con los cadetes de la Fuerza Aérea Brasileña, aviadores del grupo de 2011, en un total de 60 voluntarios. Para verificar la capacidad de ejecutar múltiples tareas, se utilizó la prueba "todo al mismo tiempo" (HERCULANO-HOUZEL, 2014). Para la prueba de impulsividad, con 45 (cuarenta y cinco) voluntarios, se usó la Escala de Impulsividad de Barratt-BIS 11.

Palabras clave: Múltiples tareas. Impulsividad. Tomada de decisión. Desempeño.

\section{INTRODUÇÃO}

O homem, como ser social, é complexo em sua totalidade. Há influências na relação entre indivíduo e organização, sendo que o administrador deve levar em conta a análise do indivíduo em particular e na coletividade do ambiente organizacional.

A desinibição comportamental constitui uma incapacidade de controle pessoal em resposta a exigências da situação. O fracasso, na inibição de comportamentos, tem recebido na literatura a denominação de impulsividade.
O propósito deste trabalho é investigar a relação entre a execução de múltiplas tarefas e a impulsividade. Há relatos na literatura de que aqueles que se julgam mais aptos para a execução de múltiplas tarefas são exatamente os mais impulsivos (SABONMATSU et al., 2013). Assim, pretende-se verificar se o mesmo acontece em uma amostra de cadetes da Academia da Força Aérea Brasileira.

Estudos do comportamento humano, sob o olhar da neurociência, partem do seguinte princípio: "o que somos, fazemos, pensamos e desejamos é resultado do funcionamento do sistema nervoso e sua interação com 
o corpo". A neurociência procura entender a relação da estrutura e do funcionamento do sistema nervoso com a história de vida de cada um; a cultura, a sociedade, e a genética fazem de nós o que somos, individualmente, como seres humanos, e como animais (HERCULANOHOUZEL, 2014).

\section{REFERENCIAL TEÓRICO}

O referencial teórico se desenvolve sob os temas da cognição organizacional e das decisões rápidas.

\subsection{Cognição organizacional}

No tocante a cognição, há uma pluralidade de linguagens e conceitos que permeiam esse campo de investigação. Entre as vertentes apresentadas nos estudos de Davel e Vergara (2001), tem-se a primeira delas com a exposição confirmatória do rompimento da noção de mente cartesiana, ou seja, a mente ser totalmente separada do corpo físico. Isso significa romper com a dicotomia razão e emoção e passar a visualizar o homem como um todo, vincular os processos cognitivos e emocionais ao corpo, sendo estes considerados centrais para a racionalidade.

Rotulada de construtivista, a segunda vertente tem o homem como sujeito da história, e não apenas um expectador. Enunciado por Davel e Vergara (2001), a mente como instrumento ativo na construção do mundo configura-se uma área específica de pesquisa na psicologia social, segundo a qual a natureza social dos processos de cognição vão além do simples processamento de informações.
As organizações burocráticas consolidam-se com a perspectiva macro-orientada nos estudos organizacionais, fundamentados na sociologia, na ciência política e na economia. Segundo Hall,

o olhar macro nos estudos organizacionais parte do comportamento das pessoas se deve a fatores organizacionais. Organizações têm o poder de moldar o comportamento e as ações individuais. Elas agem, têm políticas, fazem declarações. Elas subsistem no tempo para além das pessoas que a integram. Há uma estrutura social, um sistema de regras, normas, valores e expectativas que preexistem aos indivíduos. (HALL, 1984 apud DAVEL; VERGARA, 2001, p. 96).

As organizações, cuja influência é de uma perspectiva micro-orientada, são fundamentadas na psicologia. Sua raiz está no movimento das relações humanas, que trata o conceito de organização como algo além da interação de indivíduos. Em oposição à visão anterior, no papel determinante da organização sobre os processos individuais, essa perspectiva enfatiza mais o papel do indivíduo na construção da organização. Segundo Staw e Sutton,

indivíduos autônomos posam como organização. Ações são ditas organizacionais podem ser ações individuais, sob a aparência de uma entidade impessoal. Indivíduos com poder exercem influência e controle: ao modelar decisões estratégicas; ao definir estruturas mais estáveis; ao modelar percepções de parceiros; ao modelar características do próprio grupo de pessoas. (STAW; SUTTON, 1993 apud DAVEL; VERGARA, 2001, p. 96).

O Quadro 1 apresenta uma evolução das abordagens cognitivistas a partir dos anos 90 .

Quadro 1 - Abordagens cognitivistas.

\begin{tabular}{|c|c|}
\hline Autores & Abordagens cognitivistas \\
\hline Wilpert (1995) & $\begin{array}{l}\text { Assinala o crescimento da perspectiva simbólica e construtivista. } \\
\text { Afirma ser desnecessário o conflito entre as abordagens macro e micro-orientadas. } \\
\text { Aborda o fenômeno organizacional como socialmente construído por meio da interação de } \\
\text { autores relevantes. }\end{array}$ \\
\hline Rousseau (1997) & $\begin{array}{l}\text { A organização é vista como "processo", e não como uma "entidade". } \\
\text { Organização como uma construção social. }\end{array}$ \\
\hline $\begin{array}{c}\text { Scneider e } \\
\text { Angelmar (1993) }\end{array}$ & $\begin{array}{l}\text { Articulam três níveis de análise (indivíduo, grupo e organização) e } \\
\text { três propriedades centrais da cognição: } \\
\text { - estruturas cognitivas (como o conhecimento é adquirido e utilizado); } \\
\text { - processos cognitivos (como o conhecimento é adquirido e utilizado); e } \\
\text { - estilos cognitivos (como as unidades diferenciam-se quanto às estruturas e processos de conhecimento). }\end{array}$ \\
\hline Walsh (1995) & $\begin{array}{l}\text { Os estudos cognitivos têm-se voltado para quatro níveis de análise (indivíduo, grupo, organização, indústria) } \\
\text { e as questões cognitivas (representação, uso e desenvolvimento das "estruturas de conhecimento"). } \\
\text { o desenvolvimento e mudanças nas estruturas de conhecimento; a representação ou a "estrutura do } \\
\text { conhecimento" em si, buscando descobrir, em face de estruturas específicas utilizadas por gerentes, seus } \\
\text { atributos, conteúdos específicos e como esses se estruturam; e o uso e consequências dessas estruturas, } \\
\text { ou seja, o seu impacto sobre resultados organizacionais, em seus diferentes níveis. }\end{array}$ \\
\hline
\end{tabular}

Fonte: Adaptado de Davel e Vergara (2001). 
Assim, Davel e Vergara (2001) destacam algumas características marcantes sobre o estado da arte no campo da cognição e organização.

Os estudos sobre cognição organizacional estão voltados para explorar impactos das estruturas de conhecimento na vida das pessoas, gestores e organizações. Em contrapartida é encontrado um reduzido número de pesquisas envolvendo o processamento automático, não controlado ou inconsciente.

As abordagens que integram as estruturas cognitivas e processos de cognição são raras. E ainda, é forte o pressuposto de que a cognição individual produz comportamento organizacional que pode conduzir ao entendimento de processos coletivos como somatórios de processos individuais. Tal relação requer justificativa do ato que é salientado após sua ocorrência.

Qualquer que seja a perspectiva que consolida a organização, em uma vertente micro ou macro, encontram-se tanto indivíduos, quanto processos ocorrendo na organização. Processos estes que culminam no que se convencionou chamar de decisões.

\subsection{Tipos de decisões}

Ainda que a arte da guerra e os movimentos militares tenham influenciado alguns ramos da investigação teórica a respeito das decisões, como o das pesquisas operacionais e o da logística, os principais avanços ocorreram no âmbito das organizações privadas, de interesse econômico. Tradicionalmente, tais organizações dividem as decisões em dois grandes blocos: o bloco das decisões estratégicas e o bloco das decisões operacionais e táticas.

No bloco das decisões estratégicas, o foco é sempre o horizonte temporal mais largo e a atenção aos impactos que tais decisões podem produzir na arena de competição entre as organizações. No bloco das decisões operacionais e táticas, o horizonte passa a ser de prazo mais curto e a atenção se volta para a eficiência e a produtividade das operações.

Com o advento da economia comportamental, novos atores passaram a investigar as decisões - agora com maior foco no comportamento humano - sob o prisma da psicologia cognitiva. Em função disso, as decisões passaram a ser analisadas sob novo prisma (ou bloco): (i) as decisões racionais, tomadas em situações planejadas, envolvendo apostas de baixo risco, em ambientes de baixa pressão em termos de tempo de decisão, e apoiadas principalmente na matemática e nos postulados da lógica; e (ii) as decisões intuitivas, tomadas em situações dinâmicas, de escassez de tempo de decisão, e com pouco uso do recurso da deliberação lógica.

Em poucas palavras, decisões estruturadas e decisões rápidas. As decisões rápidas, de caráter mais intuitivo, foram classificadas como decisões baseadas no sistema 1 de pensamento e deliberações. Já as decisões estruturadas foram classificadas como decisões baseadas no sistema 2 de pensamento e deliberações (BAZERMAN, 2006).

Assim, convencionou-se que as decisões do tipo 1 seriam mais dependentes do repertório de experiências individuais, que habilitariam o tomador de decisão ao melhor uso de seu sentido de circunstancialidade e subjetividade, e as decisões do tipo 2 seriam mais dependentes do uso da lógica matemática e instrumental. Na pilotagem militar, dependendo de cada situação e etapa do voo, as decisões dos pilotos podem demandar um ou outro sistema, ou até mesmo a conjugação dos dois sistemas de pensamento e decisão.

No âmbito do presente trabalho, o interesse recai sobre as decisões mais rápidas em ambiente dinâmico, como aquele que caracteriza o voo. Outra razão para essa iluminação das questões relacionadas a decisões mais rápidas e intuitivas justifica-se pelo tema central deste trabalho, que explora as características da impulsividade quando no gerenciamento de múltiplas tarefas.

\section{METODOLOGIA}

Como já foi dito, o objetivo deste trabalho é verificar se, em uma amostra de cadetes da Academia da Força Aérea, a habilidade para o gerenciamento de múltiplas tarefas está correlacionada à impulsividade desses jovens. A pesquisa contou com um total de 45 (quarenta e cinco) voluntários $(\mathrm{N}=45$ ).

Para a verificação da habilidade em gerenciamento de múltiplas tarefas foram utilizados dois instrumentos: $\mathrm{O}$ software TAPMIL e o jogo "tudo ao mesmo tempo" (TMT) que foi disponibilizado por uma equipe de neurocientistas coordenados por Suzana Herculano-Houzel. Nesse jogo, cujo objetivo é obter maior número de pontos e, consequentemente, controlar um maior número de tarefas, aumentam-se, gradativamente, as tarefas à medida que o jogador muda de estágio.

Para a verificação da impulsividade foi aplicado o instrumento BIS-11, em que se utilizou a Escala de Impulsividade de Barratt - BIS 11. O instrumento consta de 30 (trinta) questões, sendo assinalada pelo respondente apenas uma entre as seguintes alternativas: (1) raramente ou nunca; (2) de vez em quando; (3) com frequência; (4) quase sempre ou sempre. A tabulação dos dados seguiu a pontuação para as questões que vão de 1 a $4(1,2,3,4)$. Nas questões $1,7,8,9,10,12,13,15,20,29$ e 30 , considerouse a ordem inversa $(4,3,2,1)$.

A despeito das diferenças culturais relacionadas a conceitos relativos aos diferentes tipos de impulsividade, o modelo de Barratt - BIS 11 tem sido adaptado com sucesso para diferentes culturas, o que evidencia sua validade transcultural. Dada a importância do modelo proposto por Barratt - BIS 11 para estudos sobre a impulsividade, considera-se que o desenvolvimento de uma versão brasileira da escala é de fundamental importância para a prática clínica e para a realização de estudos sobre o tema (MALLOY-DINIZ et al., 2010, p. 104).

Patton etal. (1995) definem a versão atual do instrumento, realizada por uma análise de componentes principais em BIS-11. As notas recolhidas a partir de uma amostra de 248 (duzentos e quarenta e oito) pacientes psiquiátricos internados e 412 (quatrocentos e doze) estudantes universitários. A análise fatorial, a partir desses casos, revelou três fatores de segunda ordem, aqui denominados secundários, e seis fatores oblíquos de primeira ordem, ou primários. O Quadro 2 mostra a relação entre a primeira e a segunda ordem, bem como as questões do BIS-11 nos fatores. 
Quadro 2 - Fatores primários e secundários da BIS 11.

\begin{tabular}{|c|c|c|c|c|}
\hline \multicolumn{5}{|c|}{ Escala de Impulsividade de Barratt - BIS 11} \\
\hline $\begin{array}{c}\text { Fatores } \\
\text { secundários }\end{array}$ & & $\begin{array}{l}\text { Fatores } \\
\text { primários }\end{array}$ & $\begin{array}{l}\mathrm{N}^{\circ} \text { de } \\
\text { itens }\end{array}$ & $\begin{array}{l}\text { Itens que contribuem para } \\
\text { cada subescala }\end{array}$ \\
\hline \multirow{2}{*}{$\begin{array}{l}\text { Impulsividade } \\
\text { Atencional }\end{array}$} & \multirow{2}{*}{$\begin{array}{l}\text { Impulsividade relacionada a tomada de } \\
\text { decisão rápida }\end{array}$} & Atenção & 5 & $5,9 *, 11,20 *, 28$ \\
\hline & & $\begin{array}{c}\text { Instabilidade } \\
\text { cognitiva }\end{array}$ & 3 & $6,24,26$ \\
\hline \multirow{2}{*}{$\begin{array}{l}\text { Impulsividade } \\
\text { Motora }\end{array}$} & \multirow{2}{*}{$\begin{array}{l}\text { A impulsividade motora está relacionada } \\
\text { à não inibição de respostas incoerentes } \\
\text { com o contexto }\end{array}$} & Motor & 7 & $2,3,4,17,19,22,25$ \\
\hline & & Perseverança & 4 & $16,21,23,30^{*}$ \\
\hline \multirow{2}{*}{$\begin{array}{l}\text { Impulsividade } \\
\text { por não } \\
\text { planejamento }\end{array}$} & \multirow{2}{*}{$\begin{array}{l}\text { Engloba comportamentos orientados } \\
\text { para o presente }\end{array}$} & Autocontrole & 6 & $1 *, 7^{*}, 8^{*}, 12^{*}, 13^{*}, 14$ \\
\hline & & $\begin{array}{c}\text { Complexidade } \\
\text { cognitiva }\end{array}$ & 5 & $10^{*}, 15^{*}, 18,27,29 *$ \\
\hline
\end{tabular}

Fonte: $\mathrm{O}$ autor.

Enquanto muitos manuscritos acadêmicos relatam apenas a pontuação total, é recomendado que, pelo menos, o segundo fator de ordem seja relatado para explicar a sua contribuição individual para a relação que está sendo testada (PATTOON et al., 1995).

Os resultados podem variar de 30 (trinta) a 120 (cento e vinte) pontos. Para o caso de 30 (trinta) pontos, o respondente que, nas questões normais, optar pela alternativa "raramente ou nunca" e, nas questões invertidas, optar pela alternativa "quase sempre/sempre", receberá 1 (um) ponto por questão. Para o caso de 120 (cento e vinte) pontos, o respondente que optar pela alternativa inversa ao caso anterior receberá, assim, 4 (quatro) pontos por questão.

Para análise da impulsividade, os resultados que consideram os menos impulsivos dizem respeito àqueles que se aproximaram de 30 (trinta) e os mais impulsivos, àqueles que se aproximaram de 120 (cento e vinte) pontos. Nesta pesquisa, foram considerados menos impulsivos os voluntários com pontuação no primeiro quartil e, mais impulsivos, os situados no terceiro quartil, ou seja, os extremos da amostra.

Em uma segunda etapa da pesquisa, tabularamse os dados com a inclusão da pontuação obtida no teste TAPMIL e as notas do Esquadrão de Instrução Aérea (EIA), local onde os cadetes recebem a instrução prática de voo. Vale lembrar que o teste TAPMIL avalia seis fatores, havendo-se, para esta pesquisa, considerado apenas o fator capacidade para a execução de múltiplas tarefas.
De posse das informações foram realizadas análises descritivas (média, desvio padrão, variância, máximo, mínimo, amplitude, entre outras). Para complementar, verificou-se o coeficiente de variação das variáveis em estudo.

Com os dados agrupados aos pares foi verificado o coeficiente de correlação.

A seguir, no tópico 4, tabelas 1 a 7, são apresentados os resultados.

\section{RESULTADOS}

\subsection{Análise descritiva dos testes TMT e BIS}

Tabela 1 - TMT.

\begin{tabular}{cc}
\hline & TMT \\
\hline Média & 93,53333 \\
Erro padrão & 5,495232 \\
Mediana & 88 \\
Moda & 136 \\
Desvio padrão & 36,86314 \\
Variância da amostra & 1358,891 \\
Curtose & 0,162757 \\
Assimetria & 0,73699 \\
Intervalo & 158 \\
Mínimo & 40 \\
Máximo & 198 \\
Soma & 4209 \\
Contagem & 45 \\
\hline
\end{tabular}

Fonte: $\mathrm{O}$ autor. 
Tabela 2 - BIS.

\begin{tabular}{cc}
\hline & BIS \\
\hline Média & 61,26667 \\
Erro padrão & 1,664362 \\
Mediana & 61 \\
Moda & 66 \\
Desvio padrão & 11,16488 \\
Variância da amostra & 124,6545 \\
Curtose & $-0,53319$ \\
Assimetria & 0,236712 \\
Intervalo & 45 \\
Mínimo & 42 \\
Máximo & 87 \\
Soma & 2757 \\
Contagem & 45 \\
\hline
\end{tabular}

Fonte: O autor.

O coeficiente de variação do BIS é de 18,22\% e do TMT de 39,41\%, o que significa que, abaixo de 30\%, a amostra é considerada homogênea e, acima desse percentual, considerada heterogênea.

Tabela 3 - TAPMIL.

TAPMIL

\begin{tabular}{cc}
\hline Média & 111,0667 \\
Erro padrão & 2,490061 \\
Mediana & 109 \\
Moda & 98 \\
Desvio padrão & 16,70384 \\
Variância da amostra & 279,0182 \\
Curtose & $-0,00887$ \\
Assimetria & $-0,12804$ \\
Intervalo & 82 \\
Mínimo & 64 \\
Máximo & 146 \\
Soma & 4998 \\
Contagem & 45 \\
\hline
\end{tabular}

Fonte: $\mathrm{O}$ autor.
Tabela 4 - EIA.

\begin{tabular}{cc}
\hline & EIA \\
\hline Média & 4,374933 \\
Erro padrão & 0,029532 \\
Mediana & 4,343 \\
Moda & 4,257 \\
Desvio padrão & 0,198105 \\
Variância da amostra & 0,039245 \\
Curtose & $-0,42877$ \\
Assimetria & 0,516116 \\
Intervalo & 0,8 \\
Mínimo & 4,029 \\
Máximo & 4,829 \\
Soma & 196,872 \\
Contagem & 45 \\
\hline
\end{tabular}

Fonte: $\mathrm{O}$ autor.

O coeficiente de variação do TAPMIL é de 15,04\% e do EIA, de 4,53\%, ambos abaixo de $30 \%$, o que significa homogeneidade dos dados.

\subsection{Coeficiente de correlação}

Neste subtópico são apresentados os coeficientes dos testes TMT, TAPMIL e a nota do EIA em relação ao teste BIS (impulsividade).

Tabela 5 - Correlação 1.

\begin{tabular}{ccc}
\hline \multicolumn{3}{c}{ Correlação 1 } \\
\hline BIS & BIS & TMT \\
TMT & 1 & 1 \\
\hline
\end{tabular}

Fonte: $\mathrm{O}$ autor.

Tabela 6 - Correlação 2.

\begin{tabular}{ccc}
\hline \multicolumn{3}{c}{ Correlação 2 } \\
\hline BIS & BIS & TAPMIL \\
TAPMIL & 1 & 1 \\
\hline
\end{tabular}

Fonte: $\mathrm{O}$ autor.

Tabela 7 - Correlação 3.

\begin{tabular}{ccc}
\hline \multicolumn{3}{c}{ Correlação 3 } \\
\hline BIS & BIS & EIA \\
EIA & 1 & 1 \\
\hline
\end{tabular}

Fonte: $\mathrm{O}$ autor. 
Verifica-se que não há correlação da impulsividade com os outros testes e, também, que sua relação é inversa. Ou seja, a conclusão a que se chega com a análise dos dados dos testes TMT, BIS 11, TAPMIL e notas no EIA é de que os cadetes mais hábeis em gerenciar múltiplas tarefas, assim como os mais hábeis em voo, não são aqueles que apresentam a maior impulsividade.

Essa conclusão é bastante alvissareira, pois a impulsividade é uma característica bastante indesejável no meio militar, sobretudo em se tratando da aviação militar.

\section{CONSIDERAÇÕES FINAIS}

O presente estudo partiu do interesse em verificar se a variável impulsividade interfere na execução de múltiplas tarefas e se a mesma afeta o desempenho em voo. A pesquisa de Araújo et al. (2009) concluiu que a impulsividade está associada ao comportamento de risco no trânsito, correr por aventura e infrações.

Do que foi proposto para este trabalho, não houve correlação entre a variável impulsividade e a execução de múltiplas tarefas, tampouco associação entre a impulsividade e o desempenho em voo.

\section{REFERÊNCIAS}

ARAÚJO, M. M.; MALLOY-DINIZ, L. F.; ROCHA, F. L. Impulsividade e acidentes de trânsito. Revista de Psiquiatria Clínica, v. 36, n. 2, p. 60-68, 2009.

BAZERMAN, M. H. Judgment in managerial decision making. 6th ed. Hoboken: J. Wiley \& Sons, 2006.

COSTA, M. P. Estudo de normatização e validação do teste de aptidão para a pilotagem militar. 2010. Dissertação (Mestrado) - Instituto de Psicologia, Universidade de São Paulo, 2010.

DAVEL, E.; VERGARA, S. C. (Org.). Gestão com pessoas e subjetividade. São Paulo: Atlas, 2001.

FÁVERO, L. P. et al. Análise de Dados: modelagem multivariada para tomada de decisões. Rio de Janeiro: Elsevier, 2009.

KLEIN, G. Sources of power: how people make decisions. Cambridge: MIT, 2000.

MALLOY-DINIZ, L. F. et al. Impulsiveness scale (BIS-11) para aplicação em adultos brasileiros.
Jornal Brasileiro de Psiquiatria, Rio de Janeiro, v. 59, n. 2, p. 99-105, 2010.

HERCULANO-HOUZEL, S. O cérebro nosso de cada dia. Rio de Janeiro: FAPERJ: CNPQ, 2000. Disponível em:< http://www.cerebronosso.bio.br/guiabsico-de-neurocincia>. Acesso em: 5 mar 2014.

PATTON, J. H.; STANFORD, M. S.; BARRATT, E.S. BIS-11 instrument reference. Factor structure of the Barratt impulsiveness scale. Journal of Clinical Psychology, v. 51, n. 6, p. 768-774. Disponível em: <http://www.impulsivity.org/measurement/bis11>. Acesso em: 25 mar. 2014.

SANBONMATSU, D. M et al. Who multi-tasks and why? Multi-tasking ability, perceived multi-tasking ability, impulsivity, and sensation seeking. PSO ONE, v. 8, n. 1, jan. 2013.

SILVA, L. M. A.; LUCAS, L. A.; COSTA, M. P. Analysis of decision making processo flight instructors at the Brazilian Air Force Academy. In: INTERNATIONAL CONFERENCE ON NATURALISTIC DECISION MAKING, 9., 2009, London. Proceedings of ... London: [s .n.], 2009. 